\title{
Ezetimibe in the balance: can cholesterol-lowering drugs alone be an effective therapy for NAFLD?
}

\author{
Giovanni Musso
}

Received: 10 January 2014 / Accepted: 27 January 2014 / Published online: 20 February 2014

(C) Springer-Verlag Berlin Heidelberg 2014

Keywords Cholesterol absorption $\cdot$ NAFLD $\cdot$ NASH . NPC1L1

$\begin{array}{ll}\text { Abbreviations } \\ \text { ABCA1 } & \text { ATP-binding cassette transporter A1 } \\ \text { GLP-1 } & \text { Glucagon-like peptide-1 } \\ \text { HSC } & \text { Hepatic stellate cell } \\ \text { LOX-1 } & \text { Lectin-like oxidised LDL receptor-1 } \\ \text { LPS } & \text { Lipopolysaccharide } \\ \text { LXR } & \text { Liver X receptor } \\ \text { NAFLD } & \text { Non-alcoholic fatty liver disease } \\ \text { NASH } & \text { Non-alcoholic steatohepatitis } \\ \text { NPC1L1 } & \text { Niemann-Pick C1 like 1 } \\ \text { oxLDL } & \text { Oxidised LDL } \\ \text { SREBF } & \text { Sterol regulatory element binding factor } \\ \text { SREBP } & \text { Sterol regulatory element binding protein } \\ \text { TGF } & \text { Transforming growth factor } \\ \text { TLR-4 } & \text { Toll-like receptor-4 } \\ \text { TNF } & \text { Tumor necrosis factor }\end{array}$

Non-alcoholic fatty liver disease (NAFLD) affects $30 \%$ of the general adult population and $70-80 \%$ of diabetic and obese patients [1]. NAFLD confers a 1.8-fold higher mortality, largely accounted for by liver-related complications occurring in non-alcoholic steatohepatitis (NASH), the progressive form of NAFLD; accordingly, the frequency of NASH as the primary indication for liver transplantation increased from $1.2 \%$ to $9.7 \%$ in the last decade, becoming the third most common indication for liver transplantation in Western countries [2]. In addition to liver-related complications,

G. Musso $(\bowtie)$

Gradenigo Hospital, Turin, Corso Regina Margherita 8, 10132 Turin, Italy

e-mail: giovanni_musso@yahoo.it
NAFLD is also an emerging risk factor for diabetes and cardiovascular disease [3].

There is no established treatment for NASH: lifestyleinduced weight loss is effective in less than $50 \%$ of individuals and the long-term safety of pioglitazone and vitamin E, suggested as possible therapeutic options by current guidelines [1], is far from being demonstrated; furthermore, there is no established treatment able to reverse hepatic fibrosis, the strongest predictor of future development of cirrhosis [4].

The role of non-esterified cholesterol accumulation in the pathogenesis of liver injury, and the potential therapeutic implication of cholesterol modulation in NASH, is attracting considerable interest [5]. The randomised trial by Takeshita and colleagues [6] provides a unique, thorough insight into the impact of selective cholesterol absorption inhibition on liver disease, and glucose and lipid metabolism in NAFLD. The main findings of this study are as follows:

1. In 32 biopsy-diagnosed NAFLD patients, treatment for 6 months with the Niemann-Pick C1-like 1 (NPC1L1) inhibitor ezetimibe ameliorated histological features of fibrosis and hepatocyte ballooning, while there was no significant difference in steatosis, inflammation or NAFLD activity score (NAS) compared with controls (see electronic supplementary material [ESM] Table 2 of the Takeshita et al article [6]).

2. Ezetimibe treatment was associated with an increase in $\mathrm{HbA}_{1 \mathrm{c}}$ and a worsening in whole-body, muscle and adipose tissue insulin sensitivity (the latter with borderline significance), while plasma pro-/anti-inflammatory markers and cytokines, including C-reactive protein, TNF- $\alpha$, leptin, plasminogen activator inhibitor (PAI)-1 and adiponectin were unaffected by treatment (Table 2 of the article [6]).

3. Ezetimibe treatment was associated with hepatic downregulation of genes involved in skeletal muscle development, cell adhesion and long chain NEFA $\beta$-oxidation 
(including the rate-limiting enzyme, carnitine palmitoyltransferase 1A) and with upregulation of genes involved in cholesterol biosynthesis (including sterol regulatory binding factor [SREBF]-2) and immune response/ phagocytosis (Table 3 of the article [6]).

4. These changes were accompanied by a significant increase in the hepatic fraction of several saturated fatty acids, including margaric (C17:0) and stearic (C18:0) acids (Table 6 of the article [6]).

\section{What messages can be drawn from this small, proof-of-concept study?}

The first, clinically relevant message is that inhibition of cholesterol absorption may ameliorate hepatic fibrosis in $\mathrm{NASH}$, independently of any improvement in systemic inflammation or insulin sensitivity. A major limitation of this trial is the small study size and, more importantly, the limited number of participants with the progressive form of NAFLD, NASH (eight patients with NASH of 23 NAFLD patients overall). This implies that, when we analyse the effect of treatment as a dichotomous variable (improvement vs no improvement in fibrosis), there is no significant difference between arms (4/16 patients with improved fibrosis stage following ezetimibe compared with $1 / 12$ in controls, $p=0.136$ with $\chi^{2}$ test). While the impact on liver fibrosis needs further confirmation in larger studies, given the small number of patients included, the findings of this trial corroborate recent results from experimental models, suggesting that hepatic accumulation of non-triacylglycerol toxic lipid species (non-esterified cholesterol, saturated fatty acids, ceramides and diacylglycerols) triggers endoplasmic reticulum stress, mitochondrial dysfunction and oxidative stress, promoting liver injury, steatohepatitis and fibrosis [5, 7-10]. Several putative mechanisms linking cholesterol accumulation to hepatic stellate cell (HSC) activation and fibrogenesis have been demonstrated experimentally and reviewed elsewhere [5], including enhanced toll-like receptor (TLR)-4 pathway activation, which sensitises HSCs to the key fibrogenic factor transforming growth factor (TGF)- $\beta 1[10]$, and reduces AMP-activated protein kinase- $\alpha$ (AMPK) activation [11] (Fig. 1). On this premise, whole-body and hepatic cholesterol pool depletion through inhibition of NPC1L1 in enterocytes and hepatocytes may partly explain the benefits for liver fibrosis observed in this study. However, several issues regarding the interaction between cholesterol metabolism and hepatic fibrosis remain to be elucidated, and offer potential therapeutic targets. A major issue is that HSCs lack cholesterol biosynthetic ability and NPC1L1 has not been demonstrated in HSCs, which acquire cholesterol mainly through uptake of circulating LDLs and oxidised LDLs (oxLDLs). In NASH,
Fig. 1 (a) Interactions among HSCs and Kupffer cells in promoting cholesterol-mediated liver fibrosis in NAFLD (modified from Musso et al [5]). The ability of intracellular non-esterified cholesterol (NE chol) overload to directly activate HSCs and mechanisms underlying non-esterified cholesterol accumulation in HSCs has recently been documented in mice; this involves a TLR-4-dependent pathway and triggers hepatic fibrogenesis [7]. In HSCs the key regulatory transcription factor SREBP-2 is inappropriately activated, enhancing transcription of its downstream genes coding for LDL receptor (LDL-R) and for scavenger receptors (CD36, LOX-1) for oxLDLs. High intracellular non-esterified cholesterol levels suppress the endosomal-lysosomal pathway of degradation of TLR-4, whose membrane expression consequently increases. Upregulated TLR-4 makes HSCS more sensitive to activation by NEFA and LPS, natural ligands for TLR-4. Furthermore, TLR-4 downregulates, through the adaptor molecule myeloid differentiation primary response gene 88 (MyD88), the membrane receptor Bambi (BMP and activin membranebound inhibitor homologue), a pseudoreceptor for TGF- $\beta 1$ with negative regulatory function. The removal of this inhibitor sensitises HSCs to activation by TGF- $\beta 1$ and enables secretion of the chemotactic factors monocyte chemotactic protein-1 (MCP-1) and macrophage inflammatory protein 1B (MIP1B), which recruit circulating macrophages to the liver to become Kupffer cells. Kupffer cells accumulate non-esterified cholesterol through uptake of oxidised LDLs by scavenger receptors CD36 and SR-A and become activated, secreting proinflammatory adipokines (IL-1, TNF- $\alpha$ ) and the profibrogenic cytokine TGF- $\beta 1$, which activates HSCs in a paracrine manner, promoting hepatic fibrosis [5]. According to this model, reflecting current knowledge of cholesterol metabolism and trafficking in HSCs, ezetimibe would act indirectly on HSCs by depleting whole-body cholesterol stores and consequently oxLDLs and LDLs. (b) Interactions among adipocytes and hepatocytes in promoting cholesterolmediated liver injury in NAFLD. Intracellular cholesterol accumulation induces a series of events in adipocytes and hepatocytes, collectively promoting liver injury and NAFLD. In adipocytes, cholesterol imbalance (and specifically, plasma membrane cholesterol depletion) upregulates SREBP-2, further promoting cholesterol accumulation, and impairs insulin receptor (IR) and GLUT-4 translocation to the plasma membrane [5]. Non-esterified (NE) cholesterol accumulation in adipocytes also enhances secretion of proinflammatory cytokines, including angiotensinogen, chemerin, TNF- $\alpha$ and IL- 6 which, together with elevated plasma insulin levels, downregulation of miRNA122 and possibly functional $S R E B F 2$ gene polymorphisms, directly activate SREBP-2 in hepatocytes, disrupting the physiological negative feedback by elevated cholesterol stores. Enhanced SREBF2 transcription generates SREBP-2 protein, which upregulates hydroxymethylglutaryl-CoA reductase (HMG-CoA-R), the limiting enzyme in the cholesterol biosynthetic pathway, and LDL receptors and downregulates cholesterol excretion by scavenger receptor BI (SR-BI), eventually promoting toxic non-esterified cholesterol accumulation. The $S R E B F 2$ gene also generates miRNA33a, which further downregulates cholesterol excretion through the ABCA1 transporter, and several key enzymes involved in mitochondrial NEFA $\beta$-oxidation, aggravating cholesterol retention and promoting lipotoxic NEFA accumulation. SFA, saturated fatty acids

the uptake of circulating LDLs and oxLDLs by HSCs is increased despite intracellular cholesterol accumulation, due to disruption of physiological feedback [7, 12]. Thus, combined NPC1L1 and LDL uptake inhibition may offer additive benefits for hepatic fibrosis in NASH [13].

As well as evaluating the effects of ezetimibe on liver fibrosis, this RCT also disclosed the shortcomings of a strategy using cholesterol absorption inhibition as the only therapeutic tool in NAFLD treatment: $\mathrm{HbA}_{1 \mathrm{c}}$ and insulin resistance 


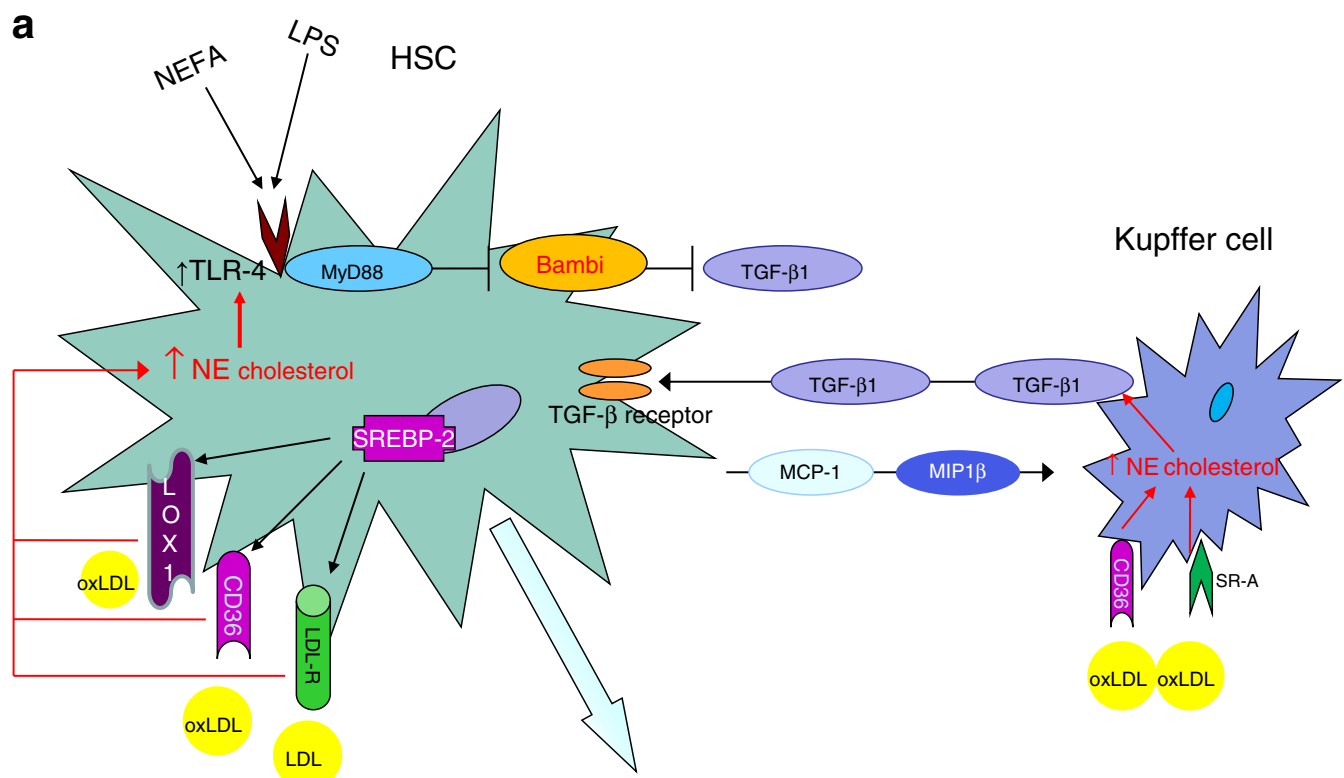

Hepatic fibrosis

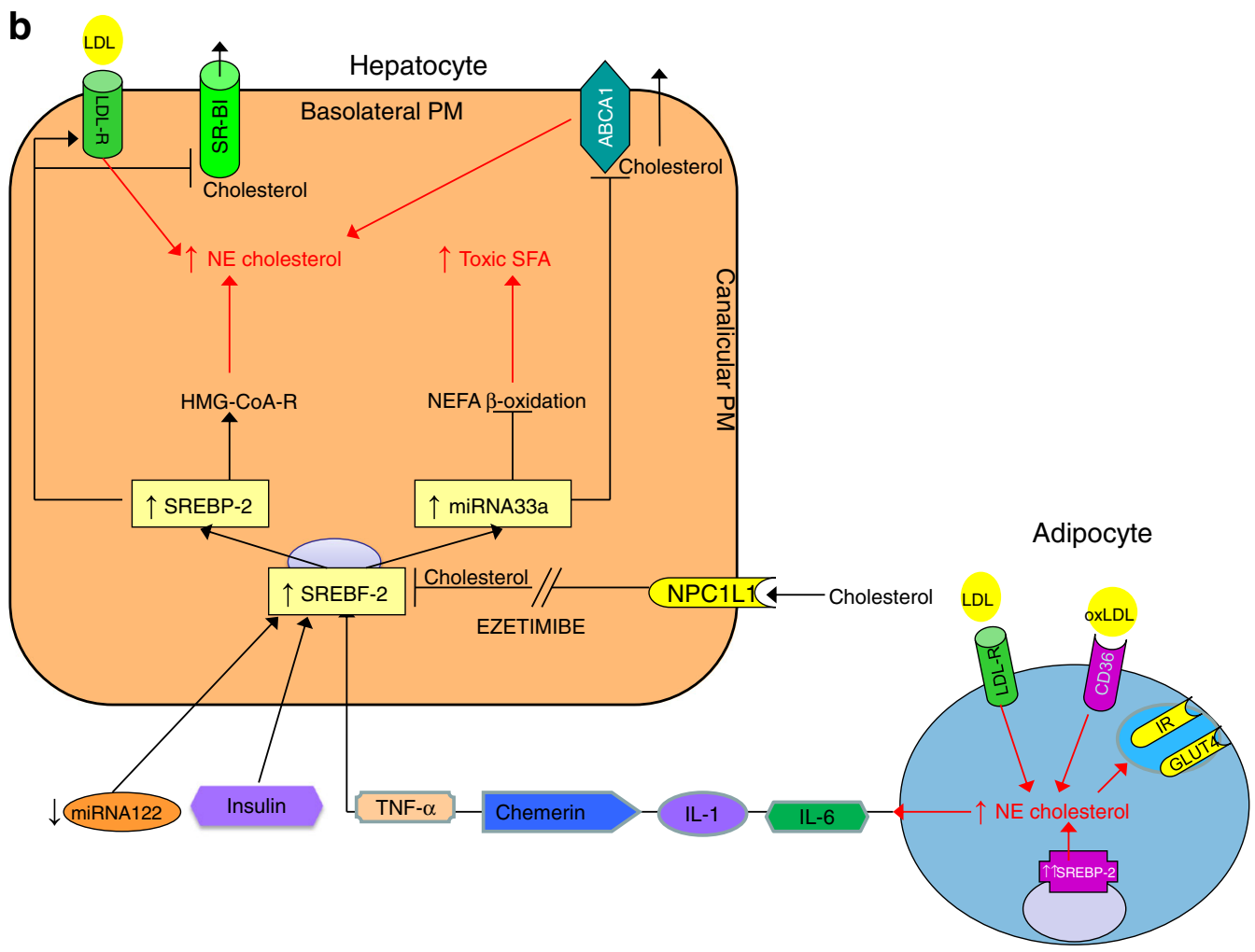

increased, hepatic steatosis was unaffected, and systemic proinflammatory and oxidative markers did not change or even tended to increase (TNF- $\alpha$, leptin, 8-isoprostanes) with ezetimibe treatment. Most of these consequences could be anticipated by understanding the changes evoked by inhibition of cholesterol uptake in different cell types, including enterocytes, hepatocytes and adipocytes, and could be adequately prevented by proper combination therapy.
The inhibition of cholesterol uptake evokes a compensatory increase in hepatic cholesterol synthesis, driven by upregulation of the gene encoding sterol regulatory element binding factor (SREBF)-2. In addition to its main product (the key regulatory nuclear transcription factor sterol regulatory element binding protein [SREBP]-2), the SREBF2 locus also generates the microRNA miRNA-33a, which is processed from an intron within the $S R E B F 2$ primary transcript [14]: 
Table 1 Potential synergisms of ezetimibe with other therapeutic strategies in NAFLD

\begin{tabular}{|c|c|c|}
\hline Agents & Mechanisms of action/rationale & Biological effects \\
\hline $\begin{array}{l}\text { Ezetimibe }+ \text { gut microbiota } \\
\text { modulation }\end{array}$ & $\begin{array}{l}\text { Intracellular non-esterified cholesterol accumulation in HSCs } \\
\text { inhibited endosomal-lysosomal TLR4 protein degradation } \\
\rightarrow \uparrow \text { TRL4 membrane expression } \rightarrow \text { sensitisation of HSCs } \\
\text { to LPS-mediated TLR4 pathway activation [7] }\end{array}$ & $\begin{array}{l}\text { Fibrogenic effects of non-esterified cholesterol diet } \\
\text { were independent of Kupffer cell activation or } \\
\text { recruitment of circulating T cells/monocytes/ } \\
\text { neutrophils into the liver }\end{array}$ \\
\hline Ezetimibe + statins & $\begin{array}{l}\downarrow \text { Cholesterol accumulation in hepatocytes, Kupffer cells and } \\
\text { HSCs [21] }\end{array}$ & $\downarrow$ hepatic inflammation and fibrosis \\
\hline Ezetimibe + curcumin & $\begin{array}{l}\text { Curcumin inhibits uptake of oxLDL through LOX-1 in HSCs } \\
{[5,10]}\end{array}$ & $\downarrow$ hepatic fibrogenesis \\
\hline $\begin{array}{l}\text { Ezetimibe + caloric (fructose) } \\
\text { restriction }\end{array}$ & $\downarrow$ ChREBP pathway activation and FAS $[25,26]$ & $\begin{array}{l}\downarrow \text { intestinal and hepatic de novo lipogenesis, } \\
\text { dyslipidaemia and insulin resistance }\end{array}$ \\
\hline Ezetimibe + GLP-1 analogues & GLP-1 analogues $\rightarrow \downarrow$ hepatic cholesterol synthesis [27] & $\downarrow$ hepatic cholesterol overload \\
\hline Ezetimibe + acarbose & $\begin{array}{l}\uparrow S R E B F 2 \text { expression } \rightarrow \uparrow \text { secretion of GLP-1 in intestinal } \\
\quad \text { enterochromaffin cells }[28,29]\end{array}$ & $\begin{array}{l}\text { Restoration of metabolic and anti-inflammatory } \\
\text { GLP-1 action }\end{array}$ \\
\hline
\end{tabular}

ChREBP, carbohydrate response element binding protein; FAS, fatty acid synthase

miRNA-33a inhibits ATP-binding cassette transporter A1 (ABCA1)-mediated cholesterol export and reduces the expression of several enzymes involved in mitochondrial fatty acid $\beta$-oxidation, thereby further promoting cellular cholesterol retention and NEFA accumulation (Fig. 1). While physiological activation of SREBP-2/miRNA33a under low cellular cholesterol conditions increases the availability of cholesterol and fatty acids to promote storage of neutral cholesteryl esters, in patients with NASH this physiological feedback is disrupted and the hepatic SREBP-2/miRNA33a axis is inappropriately activated despite cellular cholesterol overload [5], due to several reasons: direct stimulatory effects of hyperinsulinaemia $[5,15]$ and proinflammatory cytokines $[5,16]$, downregulation of hepatic miRNA122, and functional genetic polymorphisms in SREBF-2 [17] (Fig. 1). The net result is a cellular toxic non-esterified cholesterol and NEFA overload, promoting steatosis and insulin resistance in the liver, a proinflammatory pattern of adipokine secretion and enhanced release of toxic NEFA by insulin-resistant adipocytes (alterations collectively referred to as 'adipocyte dysfunction') [18]. Therefore, it is not surprising that the lack of a consistent benefit of ezetimibe on inflammation, insulin resistance and glucose homeostasis observed in this study [6] has been replicated in clinical and experimental contexts other than NAFLD [19-22], suggesting that other therapeutic approaches may be usefully combined with ezetimibe. The combination of ezetimibe with statins ensures a more consistent cellular cholesterol depletion, further reducing cholesterol toxicity, and it will be important to analyse the metabolic and liver-related effects of ezetimibe taken together with simvastatin in the NAFLD subgroup of the IMProved Reduction of Outcomes: Vytorin Efficacy International Trial (IMPROVE-IT) [23]. However, aggressive cholesterol reduction may not relieve lipotoxic NEFA accumulation, which results from reduced NEFA $\beta$-oxidation (induced by miRNA33a activation) and from stimulated de novo lipogenesis through liver
$\mathrm{X}$ receptor (LXR)- $\alpha$ /SREBP-1c axis activation: consistently, neither atorvastatin nor ezetimibe, nor their combination, improved hepatic saturated fatty acid infiltration, insulin resistance or plasma adiponectin levels in an experimental model of NASH [24].

Several other therapeutic strategies may be usefully combined with cholesterol inhibitors to enhance cholesterol unloading and improve inflammatory/metabolic variables in NAFLD. The role of dietary caloric restriction and weight loss cannot be overemphasised. while excessive cholesterol intake has also been documented in non-obese NAFLD patients [25], resembling the much higher dietary cholesterol intake $(0.2-1.25 \%)$ adopted in most experimental animal models, excessive caloric and carbohydrate consumption is common in overweight and obese NAFLD patients, which represent the vast majority of this population. Excessive dietary intake of simple carbohydrates, especially fructose, may play an important role in the pathogenesis of NAFLD-associated abnormalities [26, 27]: consistently, ezetimibe did not prevent hepatic and metabolic abnormalities induced by a high fructose diet, which triggered intestinal and hepatic de novo lipogenesis and insulin resistance independently of the cholesterol metabolic pathway [28].

The importance of caloric restriction and weight loss as an adjunct to ezetimibe emerges when comparing the study by Takeshita et al [6] with the RCT carried out by Chan et al [29]: while in the former there was no caloric restriction or weight loss in either arm, the addition of a weight-loss regimen to ezetimibe treatment significantly improved hepatic steatosis, VLDL secretion rate and systemic inflammatory markers/ cytokines compared with either weight loss or ezetimibe alone. Therefore, the synergism of caloric restriction and weight loss with cholesterol depletion could potentially fully reverse NASH-related hepatic, inflammatory and metabolic abnormalities. 
Other synergisms awaiting exploration in human trials include cholesterol depletion plus gut microbiota modulation, as it has been shown that cholesterol overload sensitises HSCs to the effect of lipopolysaccharide (LPS)/TLR-4 axis activation; and cholesterol-lowering drugs plus acarbose/glucagonlike peptide (GLP)-1 analogues, since ezetimibe stimulates GLP-1 secretion by enterochromaffin cells and, conversely, GLP-1 reduced hepatic cholesterol synthesis in experimental models [30-32].

Taken together, these issues suggest that cholesterol depletion may be a promising tool for reversing hepatic fibrosis in $\mathrm{NASH}$, but current lipid-lowering therapies may be insufficient to improve the whole cardio-metabolic profile of these patients, who are at increased risk of cardio-metabolic as well as of liver-related complications [3]. Conversely, cholesterol modulation may be a potent adjunct to other therapeutic measures that ameliorate glucose homeostasis, insulin resistance and systemic inflammation but have failed to improve hepatic fibrosis to date (Table 1). While the efficacy of these drugs warrants evaluation in large RCTs, future strategies targeting nuclear regulators of cholesterol metabolism, including farnesoid $X$ receptor (FXR), SREBP-2 and miRNA22a, are currently being evaluated in preclinical studies or phase II clinical trials and promise to open up new avenues for the development of therapeutics for $\mathrm{NASH}$ and associated disorders [5].

Duality of interest The author declares that there is no duality of interest associated with this manuscript.

Contribution statement GM was the sole contributor to this article.

\section{References}

1. Chalasani N, Younossi Z, Lavine JE et al (2012) The diagnosis and management of non-alcoholic fatty liver disease: practice guideline by the American Gastroenterological Association, American Association for the Study of Liver Diseases, and American College of Gastroenterology. Hepatology 55:2005-2023

2. Agopian VG, Kaldas FM, Hong JC et al (2012) Liver transplantation for nonalcoholic steatohepatitis: the new epidemic. Ann Surg 256: 624-633

3. Musso G, Gambino R, Cassader M, Pagano G (2011) Meta-analysis: natural history of non-alcoholic fatty liver disease (NAFLD) and diagnostic accuracy of non-invasive tests for liver disease severity. Ann Med 43:617-649

4. Musso G, Cassader M, Rosina F, Gambino R (2012) Impact of current treatments on liver disease, glucose metabolism and cardiovascular risk in non-alcoholic fatty liver disease (NAFLD): a systematic review and meta-analysis of randomised trials. Diabetologia 55: 885-904

5. Musso G, Gambino R, Cassader M (2013) Cholesterol metabolism and the pathogenesis of non-alcoholic steatohepatitis. Prog Lipid Res $52: 175-191$
6. Takeshita Y, Takamura T, Honda M et al (2013) The effects of ezetimibe on non-alcoholic fatty liver disease and glucose metabolism: a randomised controlled trial. Diabetologia. doi:10.1007/ s00125-013-3149-9

7. Egnatchik RA, Leamy AK, Noguchi Y, Shiota M, Young JD (2014) Palmitate-induced activation of mitochondrial metabolism promotes oxidative stress and apoptosis in H4IIEC3 rat hepatocytes. Metabolism 63:283-295

8. Leamy AK, Egnatchik RA, Young JD (2013) Molecular mechanisms and the role of saturated fatty acids in the progression of nonalcoholic fatty liver disease. Prog Lipid Res 52:165-174

9. Koliaki C, Roden M (2013) Hepatic energy metabolism in human diabetes mellitus, obesity and non-alcoholic fatty liver disease. Mol Cell Endocrinol 379:35-42

10. Tomita K, Teratani T, Suzuki T et al (2014) Free cholesterol accumulation in hepatic stellate cells: mechanism of liver fibrosis aggravation in nonalcoholic steatohepatitis in mice. Hepatology 59:154-169

11. Cao W, Yan L, Wang W, Zhao CY (2012) [Simvastatin inhibits activation of hepatic stellate cells and promotes activation of adenosine monophosphate-activated protein kinase]. Zhonghua Gan Zang Bing Za Zhi 20:304-309 [Article in Chinese]

12. Musso G, Cassader M, de Michieli F, Saba F, Bo S, Gambino R (2011) Effect of lectin-like oxidized LDL receptor-1 polymorphism on liver disease, glucose homeostasis, and postprandial lipoprotein metabolism in nonalcoholic steatohepatitis. Am J Clin Nutr 94:10331042

13. Kang Q, Chen A (2009) Curcumin eliminates oxidized LDL roles in activating hepatic stellate cells by suppressing gene expression of lectin-like oxidized LDL receptor-1. Lab Invest 89:1275-1290

14. Gerin I, Clerbaux LA, Haumont O et al (2010) Expression of miR-33 from an SREBP2 intron inhibits cholesterol export and fatty acid oxidation. J Biol Chem 285:33652-33661

15. Xie X, Liao H, Dang H et al (2009) Down-regulation of hepatic HNF $4 \alpha$ gene expression during hyperinsulinemia via SREBPs. Mol Endocrinol 23:434-443

16. Ye Q, Chen Y, Lei H et al (2009) Inflammatory stress increases unmodified LDL uptake via LDL receptor: an alternative pathway for macrophage foam-cell formation. Inflamm Res 58:809-818

17. Musso G, Cassader M, Bo S, de Michieli F, Gambino R (2013) Sterol regulatory element-binding factor 2 (SREBF-2) predicts 7-year NAFLD incidence and severity of liver disease and lipoprotein and glucose dysmetabolism. Diabetes 62:1109-1120

18. Musso G, Cassader M, de Michieli F, Rosina F, Orlandi F, Gambino R (2012) Nonalcoholic steatohepatitis versus steatosis: adipose tissue insulin resistance and dysfunctional response to fat ingestion predict liver injury and altered glucose and lipoprotein metabolism. Hepatology 56:933-942

19. Umemoto T, Subramanian S, Ding $Y$ et al (2012) Inhibition of intestinal cholesterol absorption decreases atherosclerosis but not adipose tissue inflammation. J Lipid Res 53:2380-2389

20. Barbosa SP, Lins LC, Fonseca FA et al (2013) Effects of ezetimibe on markers of synthesis and absorption of cholesterol in high-risk patients with elevated C-reactive protein. Life Sci 92:845-851

21. Bracht L, Barbosa CP, Caparroz-Assef SM et al (2012) Effects of simvastatin, atorvastatin, ezetimibe, and ezetimibe + simvastatin combination on the inflammatory process and on the liver metabolic changes of arthritic rats. Fundam Clin Pharmacol 26:722-734

22. Kikuchi K, Nezu U, Inazumi K et al (2012) Double-blind randomized clinical trial of the effects of ezetimibe on postprandial hyperlipidaemia and hyperglycaemia. J Atheroscler Thromb 19:1093-1101

23. Cannon CP, Giugliano RP, Blazing MA et al (2008) Rationale and design of IMPROVE-IT (IMProved Reduction of Outcomes: Vytorin Efficacy International Trial): comparison of ezetimbe/simvastatin versus simvastatin monotherapy on cardiovascular outcomes in patients with acute coronary syndromes. Am Heart J 156:826-832 
24. Van Rooyen DM, Gan LT, Yeh MM et al (2013) Pharmacological cholesterol lowering reverses fibrotic NASH in obese, diabetic mice with metabolic syndrome. J Hepatol 59:144-152

25. Yasutake K, Nakamuta M, Shima Y et al (2009) Nutritional investigation of non-obese patients with non-alcoholic fatty liver disease: the significance of dietary cholesterol. Scand J Gastroenterol 44:471477

26. Schultz A, Neil D, Aguila MB, Mandarim-de-Lacerda CA (2013) Hepatic adverse effects of fructose consumption independent of overweight/obesity. Int J Mol Sci 14:21873-21886

27. Vos MB (2014) Nutrition, nonalcoholic fatty liver disease and the microbiome: recent progress in the field. Curr Opin Lipidol 25:61-66

28. Ushio M, Nishio Y, Sekine O et al (2013) Ezetimibe prevents hepatic steatosis induced by a high-fat but not a high-fructose diet. Am J Physiol Endocrinol Metab 305:E293-E304
29. Chan DC, Watts GF, Gan SK, Ooi EM, Barrett PH (2010) Effect of ezetimibe on hepatic fat, inflammatory markers, and apolipoprotein B-100 kinetics in insulin-resistant obese subjects on a weight loss diet. Diabetes Care 33:1134-1139

30. Nakade Y, Yamamoto T, Banno F et al (2013) Effect of glucagon-like peptide-1 (GLP-1) analogue on hepatic cholesterol metabolism in nonalcoholic steatohepatitis (NASH). Gastroenterology 142(Suppl 1): S1023-S1024

31. Rudovich NN, Weickert MO, Machann J, Pfeiffer AF (2010) Combination of acarbose and ezetimibe prevents non-alcoholic fatty liver disease: a break of intestinal insulin resistance? J Hepatol 52: 952-953

32. Jeon TI, Zhu B, Larson JL, Osborne TF (2008) SREBP-2 regulates gut peptide secretion through intestinal bitter taste receptor signaling in mice. J Clin Invest 118:3693-7300 